\title{
A POSSIBILITY OF MEASUREMENT OF DISTANCE AND PROPER MOTION OF GALACTIC SOURCES BY USING A DIFFERENTIAL VLBI METHOD OF VERA
}

\author{
O. KAMEYA, T. SASAO, AND M. MIYOSHI \\ National Astronomical Observatory, Mizusawa \\ 2-12 Hoshigaoka, Mizusawa, Iwate 023, Japan \\ kameya@miz.nao.ac.jp, sasao@miz.nao.ac.jp, \\ miyoshi@miz.nao.ac.jp
}

\section{The VERA System}

National Astronomical Observatory, Japan has a plan to construct a new VLBI system named VERA ( VLBI Exploration of Radio Astrometry). The system can measure precise positions of Galactic radio sources $\left(\mathrm{H}_{2} \mathrm{O}\right.$ masers and $\mathrm{SiO}$ masers) referring to adjacent radio sources ( QSO etc.) by using a differential VLBI method (Sasao and Morimoto, 1991).

The VERA system is designed as follows:

TABLE 1. The VERA system

\begin{tabular}{ll}
\hline Antenna & about 20m diameter \\
Site & Mizusawa, Kagoshima, Ishigaki, Tsushima \\
Baseline Length & Max:2272km(Mizusawa-Ishigaki) \\
& Min:324km(Kagoshima-Tsushima) \\
Frequency & $43 \mathrm{GHz}\left(\mathrm{SiO}\right.$ maser), 22GHz( $\mathrm{H}_{2} \mathrm{O}$ maser $), 8 \mathrm{GHz}, 2 \mathrm{GHz}$ \\
Backend Rec.Speed & $1 \mathrm{Gbps}$ \\
Correlator & FX Correlator at Mitaka \\
\hline
\end{tabular}

VERA system will be operated by an Array Operational Center at Mizusawa. The recorded data tapes will be transported to Mitaka FX Correlator for correlation. The VERA antennas are designed to slew very quickly in order to perform fast-switching (10-20 sec cycle) differential VLBI observations. 


\section{Targets of VERA}

The expected positional accuracy of the VERA is about 10 microarcseconds. It means that distance of radio sources in our Galaxy can be measured via annual trigonometric parallax with the accuracy of $\mathrm{D} / 2$ percent for sources of distance of $\mathrm{D} \mathrm{kpc}$. The distance of sources can be measured less than 10 percent within the area of distance less than $20 \mathrm{kpc}$. The proper motion can be measured with the accuracy of $0.05 \mathrm{D} \mathrm{km} / \mathrm{s}$. On the other hand, radial velocity of the masers can be measured with accuracy less than $0.5 \mathrm{~km} / \mathrm{s}$ by the Doppler Effect. Thus we can measure the three dimensional velocity field in our Galaxy. It means that we can obtain both three dimensional position and velocity of the Galactic source with very small errors. And these results are free from any assumption on the motion of the Galaxy like axisymmetric rotation around the Galactic center.

There are many scientific targets of VERA by using the results of the high precision astrometry. The first one is detection of non-axisymmetric motion of $\mathrm{SiO}$ masers and $\mathrm{H}_{2} \mathrm{O}$ masers in the Galactic central region. There has been some indications of the bar structure at the Galactic central region. Detection of the non-axisymmetric motion possibly related to the barstructure would greately advance the study of the dynamics of the Galactic central region.

Second is the accurate determination of the Galactic rotation curve. Since the Galactic rotation curve is affected by dark matter in our Galaxy, measurement of the distance and proper motion of the Galactic sources is very important to infer the mass of the Galactic dark matter. Determination of the rotaion curve of the outer part of the Solar circle has been very defficult because of a large amount of ammbiguity to measure distance of Galactic sources from the earth. Since VERA can measure the distance very accurately, determination of the rotation curve of the outer part will be much improved.

Third is to measure the distance and proper motion of Galactic center. $\operatorname{Sgr} A^{*}$ is the radio source which is believed to be at the Galactic center (Alberdi et al., 1993). If the source is at $8.5 \mathrm{kpc}$, then the distance and the proper motion of it can be measured with the accuracy of 4 percent and $0.4 \mathrm{~km} / \mathrm{s}$, respectively.

\section{References}

Alberdi, A., Lara, L., Marcaido, J.M., Elosegui, P., Shapiro, I.I., Cotton, W.D., Diamond, P.J., Romney, J.D., and Preston, R.A. (1993), VLBI image of $\operatorname{Sgr} A^{*}$ at $\lambda=1.35 \mathrm{~cm}$, Astron. Astrophy., 277, pp.L1-L4

Sasao, T., and Moriomoto, M. (1991), Antennacluster-antennacluster VLBI for geodesy and astrometry, in Chapmann Conference on Geodetic VLBI: Monitoring Global Change, NOAA, Tech. Rep. NOS 137 NGS49, pp.48-62 